\title{
Automated Algorithm for Generalized Tonic-Clonic Epileptic Seizure Onset Detection Based on sEMG Zero-Crossing Rate
}

\author{
Conradsen, Isa; Beniczky, Sándor; Hoppe, Karsten; Wolf, Peter; Sørensen, Helge Bjarup Dissing
}

Published in:

I E E E Transactions on Biomedical Engineering

Link to article, DOI:

10.1109/TBME.2011.2178094

Publication date:

2012

Link back to DTU Orbit

Citation (APA):

Conradsen, I., Beniczky, S., Hoppe, K., Wolf, P., \& Sørensen, H. B. D. (2012). Automated Algorithm for Generalized Tonic-Clonic Epileptic Seizure Onset Detection Based on sEMG Zero-Crossing Rate. I E E E Transactions on Biomedical Engineering, 59(2), 579-585. https://doi.org/10.1109/TBME.2011.2178094

\section{General rights}

Copyright and moral rights for the publications made accessible in the public portal are retained by the authors and/or other copyright owners and it is a condition of accessing publications that users recognise and abide by the legal requirements associated with these rights.

- Users may download and print one copy of any publication from the public portal for the purpose of private study or research.

- You may not further distribute the material or use it for any profit-making activity or commercial gain

- You may freely distribute the URL identifying the publication in the public portal 


\title{
Automated algorithm for generalised tonic-clonic epileptic seizure onset detection based on sEMG zero-crossing rate
}

\author{
Isa Conradsen, Student Member, IEEE, Sándor Beniczky, Karsten Hoppe, Peter Wolf and Helge B.D. \\ Sorensen Member, IEEE
}

\begin{abstract}
Patients are not able to call for help during a generalized tonic-clonic epileptic seizure. Our objective was to develop a robust generic algorithm for automatic detection of tonic-clonic seizures, based on surface electromyography (sEMG) signals suitable for a portable device. Twenty-two seizures were analysed from 11 consecutive patients. Our method is based on a high-pass filtering with a cut-off at $150 \mathrm{~Hz}$, and monitoring a count of zero-crossings with a hysteresis of $\pm 50 \mu \mathrm{V}$. Based on data from one sEMG electrode (on the deltoid muscle) we achieved a sensitivity of $100 \%$ with a mean detection-latency of $13.7 \mathrm{~s}$, while the rate of false detection was limited to 1 false alarm per 24 hours. The overall performance of the presented generic algorithm is adequate for clinical implementation.
\end{abstract}

Index Terms-Epilepsy, seizure detection, tonic-clonic, sEMG.

\section{INTRODUCTION}

$\mathbf{E}$ PILEPSY is a neurological disorder that causes seizures due to an abnormal excessive or synchronous neural activity in the brain [1]. About $0.5-1 \%$ of the world's population suffers from this condition [2]. In spite of much progress with pharmacological, surgical and alternative treatments (ketogenic diet and vagal nerve stimulation), about $30-40 \%$ of epilepsy patients continue to have seizures [2]. For many of these patients, seizure onset is unpredictable, impairing independent living and increasing the risk of injuries, e.g. by falls or burns. As patients do not remember the seizures, many of these episodes will be unrecorded (if not observed by someone else). The lack of precise data on the frequency of seizure occurrence precludes the optimal adjustment of the treatment. Therapy resistant patients with generalized tonicclonic seizures have an increased risk of dying in connection with a seizure, especially when they live alone and the seizures occur during sleep [3], [4]. An alarm system, capable of detecting these seizures, could help the patients by alerting relatives and caretakers, whenever a seizure occurs.

I. Conradsen is with the Biomedical Department of Electrical Engineering, Technical University of Denmark, 2800 Kgs. Lyngby, Denmark, and the Danish Epilepsy Centre, 4293 Dianalund, Denmark e-mail: isaconradsen@gmail.com

S. Beniczky is with the Danish Epilepsy Centre, 4293 Dianalund, Denmark and Aarhus University Hospital, Department of Clinical Neurophysiology, 8000 Aarhus C, Denmark, P. Wolf is with the Danish Epilepsy Centre, 4293 Dianalund, Denmark, K. Hoppe is with DELTA, Denmark and H.B.D. Sorensen is with the Biomedical Department of Electrical Engineering, Technical University of Denmark, 2800 Kgs. Lyngby, Denmark.e-mail: hbs@elektro.dtu.dk
Previously we have focused on using multi-modal data, including sEMG and accelerometers for detection of epileptic seizures with motor manifestations [5], [6]. One other group has also tried to detect seizures based on a combination of accelerometers and sEMG [7]. Other authors have used electroencephalography (EEG) [8], [9], [10], electrocardiography (ECG) [11] or accelerometers [12], [13], [14], [15], [16] to develop a seizure detection system for tonic-clonic seizures. One group have even tried to discriminate tonicclonic seizures from other seizures based on accelerometers [17], [18]. Both Kramer et al. and Lockman et al. achieved promising results on detecting tonic-clonic seizures based on accelerometer data. However the seizures were detected rather late since the accelerometers were best at detecting the clonic phase of the seizures. Our aim was to obtain better results by developing a sensitive and specific algorithm that detects the seizures already in the tonic-phase (that precedes the clonic one). We chose surface electromyograms (sEMG) as our modality (signals), because there is an intensive activation of the muscles during the tonic phase. To make the system feasible (easy to wear by the patients) we aimed at using as few sensors as possible (only two channels). Furthermore we focused on keeping the algorithm computationally efficient to make an implementation of the algorithm in a portable device possible. The main aim of the study is to propose the first algorithm based on only sEMG signals for detecting epileptic generalised tonic-clonic seizures. Our hypothesis is that the information content of the sEMG is sufficient for early detection of tonic-clonic seizures. A preliminary version of this work has been reported[19].

\section{RECORDINGS}

Sixty consecutive patients admitted to the Epilepsy Monitoring Unit at the Danish Epilepsy Center in Dianalund, Denmark for diagnostic reasons, who had a history of tonic-clonic seizures in the referral were included. Eleven patients had tonic-clonic seizures. The rest of the patients had seizures other than tonic-clonic or did not have epileptic seizures at all during the monitoring period. The study was approved by the regional ethics committee, and it was conducted according to the declaration of Helsinki. The recordings included EEG, video, ECG and sEMG electrodes. We used $9 \mathrm{~mm}$ silver/silver chloride sEMG electrodes placed on the deltoid and anterior tibial muscles on both sides in a monopolar setting (the active 
electrode was placed on the midpoint of the muscle belly, while the reference electrode was placed on the acromyoclavicular joint, just proximal to the insertion of the muscle). For this study we have only analysed the signals from the left deltoid and anterior tibial muscles. The admission lasted 1-4 days depending on the patient. The sEMG was sampled with a frequency of $1024 \mathrm{~Hz}$. The long-term video-EEG recordings were reviewed by a clinical neurophysiologist and an epileptologist, who marked the time-epochs containing a tonic-clonic seizure, based on visual analysis. The physician marked the start of the tonic phase, when this was unequivocal. In total we recorded 22 tonic-clonic seizures in 776 hours. The number of seizures, the demographic data and the recording time for each patient is listed in Table I. During the long term monitoring,

TABLE I

THE PATIENTS GENDER, AGE, THE AMOUNT OF SEIZURES AND THE LENGTH OF THE FILES.

\begin{tabular}{c||c|c|c|c} 
Patient & Gender & Age & \# seizures & File length [h] \\
\hline \hline 1 & F & 23 & 1 & $15.9-25.3$ \\
2 & F & 26 & 2 & $92.5-95.2$ \\
3 & M & 39 & 1 & $89.4-93.4$ \\
4 & M & 25 & 1 & 46.6 \\
5 & M & 62 & 2 & $89.9-95.5$ \\
6 & M & 38 & 1 & $90.9-95.2$ \\
7 & M & 19 & 1 & $91.5-94.3$ \\
8 & M & 55 & 3 & $12.4-16.2$ \\
9 & F & 30 & 4 & 37.2 \\
10 & M & 11 & 3 & 88.1 \\
11 & M & 26 & 3 & 89.0 \\
\hline
\end{tabular}

trained neurophysiology technicians monitored the recordings to make sure that data showed EMG activity and not noise, which would imply a loose connection (high impedance). It happened that the sEMG electrodes were accidentally detached in some patients. In these cases the technicians corrected this as soon as possible. The epochs with detached/loose electrodes were excluded from the analysis, but in total more than $96 \%$ of the data was used, making it reasonable to look at the algorithm working both at night and during the day. Since some periods were excluded, the time lengths were not exactly the same for the two muscles, therefore different time length are given in Table I.

\section{METHODS}

The methods section is divided into two sections: the feature extraction and the detection approach, respectively.

\section{A. Feature Extraction}

In a previous study we analyzed the similarities and differences between sEMG signals from real epileptic seizures and sEMG signals from simulated seizures [20]. We showed, that real seizures in contrast to normal activity had a large proportion of data in the frequency band above $100 \mathrm{~Hz}$. In this study a visual evaluation of all seizures from the 11 patients revealed that the differences between seizures and normal activities, were even more pronounced, when processed with a high-pass filter with a cut-off frequency at $150 \mathrm{~Hz}$. The high pass filtering furthermore ensures, that a larger amount of the artifacts will be removed. We have used a Butterworth filter
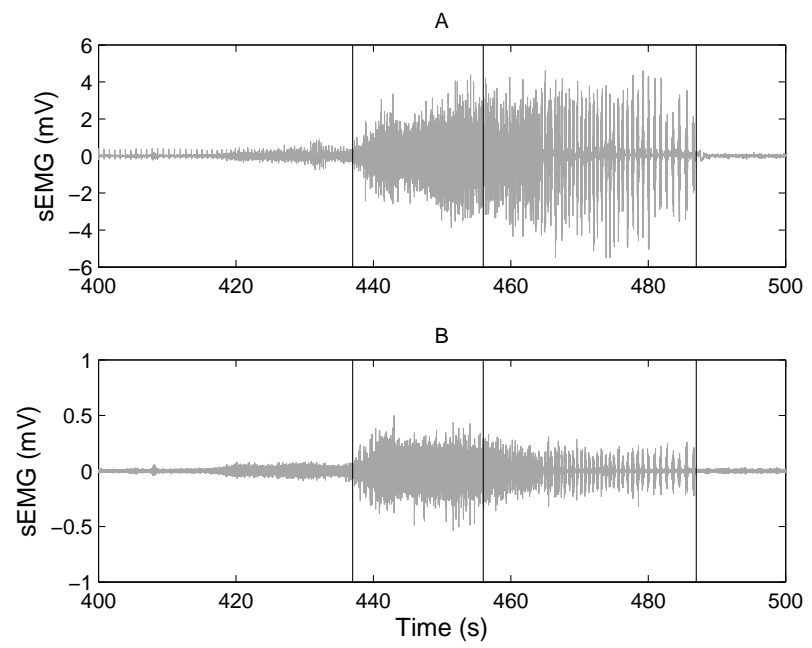

Fig. 1. Figure 'A' shows the seizure (with surrounding activity) before filtering and figure 'B' the signal after filtering. The right and left black vertical lines denote the beginning and end of the generalised tonic-clonic seizure, whereas the middle black vertical line denotes the start of the clonic phase, marked by the physician. The data is from the left deltoid.

with an order of 20 for the filtering, where the group delay is ensured to be linear in the frequency band of interest. A seizure from a representative patient, is shown in Fig. 1 before and after filtering.

Our previous study [20] on the sEMG signals during real and simulated seizures showed that simple features are able to distinguish between the two groups. Therefore we chose to focus this study on finding a simple and computationally efficient feature, that would be able to discriminate tonicclonic seizures from normal activity. The final method is meant to be used in a seizure detection system and it is therefore important to capture the seizures soon after the onset. Since the seizures are started by a tonic phase, we searched for a feature to discriminate this part of the seizure from normal activities. In our previous study [20] we found that the epileptic (generalised tonic and the tonic phase of the generalised tonic-clonic) seizures contained a larger proportion of higher frequencies than normal activities. We have therefore chosen to focus our feature choice on the frequency domain, since this might distinguish both types of seizures from normal activities (though we only focus on one type in this study). The authors of [12], [13], who have used accelerometer data, found algorithms to distinguish the clonic part from normal activities. These methods seem to perform well, but have longer latencies, because the clonic phase only comes after the tonic phase of the seizure. We chose a simple measure for the instantaneous frequency through the zero-crossing compared to the power spectrum (used in [20]), since it is more convenient for implementation in a portable detection device. Previously other groups [21], [22] have used zero-crossing for prediction of epileptic seizures based on EEG. Since we wanted our algorithm to focus only on actual sEMG data, we decided to count only those zero-crossing, which extended above and below a hysteresis. This ensured that the actual zero-crossing count would not be affected by noise. From a 
quantitative inspection of our data, we found background noise with a standard deviation (SD) as high as $15 \mu \mathrm{V}$, so to ensure that the zero-crossing only operates outside the noise region, we chose to include a hysteresis of $\pm 50 \mu \mathrm{V}$, corresponding to $3 \mathrm{SD} \approx 50 \mu \mathrm{V}$. A zero-crossing is then only counted when the signal peaks preceding and following it exceed $50 \mu \mathrm{V}$ and $-50 \mu \mathrm{V}$ respectively. So if the signal starts by rising above $50 \mu \mathrm{V}$ one count is set when the signal goes below $-50 \mu \mathrm{V}$ and another count is set, when the signal again is above $50 \mu \mathrm{V}$ and so on. We found that when applying the zero-crossing method with a hysteresis of $\pm 50 \mu \mathrm{V}$ on the filtered data, the number of crossings was high throughout the entire tonic phase, see Fig. 2. The count of zero-crossings is seen to decrease at the end of the tonic phase and throughout the clonic phase. This decrease is however caused by the clonic phase consisting of alternating periods with high activity and no activity at all. We evaluated the count of zero-crossings with a smaller window size and found that the count is as high in the active clonic phases as in the tonic phase, so the reason for the decrease in the number of counts is that the window includes both the active periods and the periods with no activity in the clonic phase.

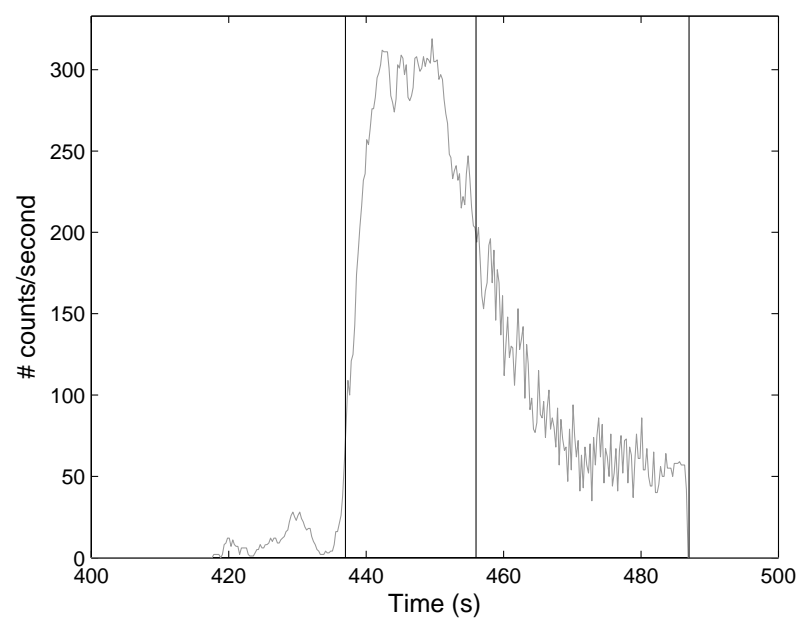

Fig. 2. The number of zero-crossings in windows of 1 second as a function of the time for the filtered data in Fig. 1 (one seizure from a representative patient). It is clearly seen that the number of zero-crossings rises fast at the start of the tonic phase, stays high throughout the tonic phase and drops at the beginning of the clonic phase. The right and left black vertical lines denote the beginning and end of the generalised tonic-clonic seizure, whereas the middle black vertical line denotes the beginning of the clonic phase.

\section{B. Detection Approach}

Although many more parameters could be varied to make the algorithm more advanced, in our search for the optimal method to classify the data into tonic-clonic seizures or normal activity, we chose to vary two parameters. The first one is the number of zero-crossings in a given window (called the threshold) and the second one is the number of succeeding windows, where the number of zero-crossings exceeds the threshold, needed to finally classify a seizure. As in one of our previous studies, we chose to use a window of 1 second
[6]. In this study we opted for an overlap of $75 \%$ for the windows. These two values, length and overlap of windows, were chosen based on a visual inspection of the feature-plot (see example in Fig. 2) for all seizures. Furthermore, this inspection showed that the maximum number of zero-crossings during the tonic phase of the seizures was about 255 counts if all seizures were to be detected. We varied the threshold from 200 (180 for anterior tibial muscle) to 300, with an interval of 5 counts between (180) 200 and 240 and between 260 and 300, whereas we had an interval of one count from 241-259. When seeking to avoid too many false detections and at the same time ensuring a sufficiently short latency, the band of properly chosen numbers of windows to make a seizure detection is most likely narrow. We therefore varied the number of windows to make a seizure detection from 2 to 30 , where two windows correspond to a minimum delay of 1.25 seconds and 30 to a minimum delay of 8.25 seconds, to ensure that all possible solutions are tested. The number of windows was varied with intervals of two between 2 and 10 and between 20 and 30, whereas it was varied with intervals of one between 11 and 19. The beginning of the tonic-clonic seizures were marked by clinical neurophysiologist and epileptologist by a visual evaluation of data, as this is more reliable than any automated method, thus far. However the exact start-time was sometimes uncertain. In these cases we opted for marking the clinical time-point that unequivocally showed the onset of the tonic phase. Thus, in theory this marking might come a few seconds later than the real seizure-onset. We therefore added an equation in our interpretation of the results which changed the latency to the minimum (based on the number of windows included) if the estimated start-time turned out to be earlier than the clinical (actual) time-point (though within 100 seconds from it). For each pair of parameters three measures were calculated to evaluate the results:

- The sensitivity (SEN): the percentage of the seizures, which were classified by the algorithm.

- The false detection rate (FDR): the amount of false detections (normal activity classified as a seizure) per hour. This is a measure of the specificity.

- The latency (LAT): the time from the beginning of a seizure to the detection of that seizure.

We used a 4-fold cross validation method [23], where the 11 patients $(\mathrm{pt})$ were randomly partitioned into four subgroups (1: pt $2,6,11 ; 2$ : pt $1,4,9 ; 3$ : pt $5,7,10 ; 4:$ pt 3,8$)$, to evaluate which parameters were optimal. From the 4 subgroups one was retained for validation of the parameters, whereas the other three subgroups were used for training the optimal choice of parameters. The validation group was then used to evaluate the trained choice of parameters. The cross-validation process was repeated four times, one time with each of the four subgroups as validation group. This method ensured that all patients were used (an equal number of times) for both training and validation. The optimal parameters for each training session were chosen from a 2D-plot, which express the relationship between the sensitivity and FDR (specificity), and the latency. The plot express the mean latency (for all seizures in the training groups) on the abscissa: 


$$
\text { abscissa }=\overline{\mathrm{LAT}},
$$

and the sensitivity minus the false detection rate on the ordinate:

$$
\text { ordinate }=\left\{\begin{array}{ll}
\text { SEN }- \text { FDR, } & \text { for SEN }- \text { FDR } \geq 0 \\
0, & \text { for SEN }- \text { FDR }<0
\end{array}\right\}
$$

where SEN is the sensitivity (between 0 and 1) and FDR is the false detection rate given per hour (the FDR corresponds to the specificity). If none of the seizures for a patient are detected the latency is given the value of the maximum latency of the patients involved in the training session. The approach of plotting the sensitivity and the FDR on one axis, and the latency on the other makes it easier to search parameters that both ensures high sensitivity, low FDR (i.e. high specificity) and short latency. In Fig. 3 an example of the plot is shown for the training session of group 1-3. The point on the curves in Fig. 3 is chosen as the best trade-off between the sensitivity and the FDR and the latency in our point of view. We have prioritized a sensitivity as close to $100 \%$ as possible and at the same time as low an FDR as possible, secondly we also tried to obtain a short latency. This is because we would rather have the detections delayed by a second, than not detecting them at all. In Fig. 3 the optimal point with respect to achieving both high sensitivity and specificity would be as close to 1 as possible on the y-axis. Secondly we chose the point so as the latency would not be too large (the point being placed too far right on the $\mathrm{x}$-axis). The optimal parameters are considered not to be outliers, so that small changes in the threshold or number of windows to finalize a detection does not change drastically (e.g. the amount of seizures detected). If so another set of optimal parameters will be searched.

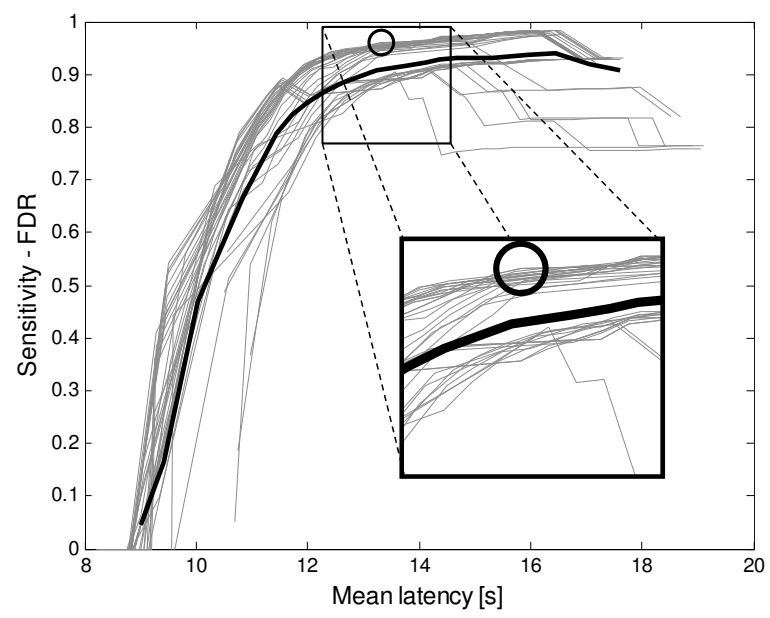

Fig. 3. Each curve shows different threshold levels and for each curve the number of windows is varied. The black circle marks the chosen point on the curves. The thicker curve highlights the mean of the curves.

The optimal choice of parameters (chosen based on a plot equal to the one shown in Fig. 3) for each of the four training sessions are given in Table II for the three training branches: deltoid muscle data alone, anterior tibial muscle data alone and the combination of data from both muscles. The parameters for the combination of the two muscles are achieved by requesting that the seizure should be visible through the features in both muscles (channels) at the same time. This combination should reduce the number of false alarms, which are only visible in one muscle. Therefore lower values are expected for the two parameters, compared to the detection being based on just one muscle.

TABLE II

THE PARAMETERS CHOSEN TROUGH THE FOUR TRAINING PHASES. \#WIN=NUMBER OF WINDOWS, TH=THRESHOLD.

\begin{tabular}{c||c|c|c|c|c|c}
\multirow{2}{*}{$\begin{array}{c}\text { Training } \\
\text { groups }\end{array}$} & \multicolumn{2}{c|}{ Deltoid } & \multicolumn{2}{c|}{ Tibialis } & \multicolumn{2}{c}{ Combined } \\
\cline { 2 - 7 } \# win & th & \# win & th & \# win & th \\
\hline \hline $1,2,3$ & 19 & 241 & 26 & 195 & 8 & 200 \\
$1,2,4$ & 15 & 253 & 28 & 195 & 8 & 195 \\
$1,3,4$ & 19 & 245 & 24 & 190 & 8 & 200 \\
$2,3,4$ & 19 & 240 & 24 & 205 & 8 & 200 \\
\hline
\end{tabular}

\section{RESUlts}

The test results for the two electrode placements (deltoid and anterior tibial) are presented in Table III together with the combined results where an alarm is generated if it is registered in both muscles at the same time. The overall results of the evaluation are very promising and suggest that it is possible to choose parameters such that the same algorithm (incl. parameters) may be used for all patients, providing a generic method for a detection system for epileptic patients with generalised tonic-clonic seizures.

When using data only from the deltoid muscle all seizures are detected with an acceptable mean latency. The latency is different however for the different patients, since not all patients have seizures which start equally abruptly and furthermore how early the muscles are recruited into the seizure varies. A visual inspection of the sEMG data compared to the video shows that the seizures for patient 9 , for whom the latency is very long, involve the deltoid muscle relatively late. Besides good sensitivity and latency, the results for the deltoid muscle alone also show a very low FDR. The mean FDR is 0.04 , which corresponds to approximately 1 in 24-hours. Most of the false detections are in the daytime, and only three were during the night $(12 \mathrm{pm}-8 \mathrm{am})$ for the results on the deltoid muscle data, see Fig. 4. This is only approximately $10 \%$ of the false alarms, so if the algorithm was implemented in a system only to be used during the night, where a surveillance system is mostly needed, the FDR would be approximately one false alarm for every tenth day.

The results for the data from the anterior tibial muscle alone are not as good as for the deltoid muscle. For the anterior tibial the mean sensitivity is $77 \%$. Only for 7 of the patients are all seizures detected, for two of the remaining three, none of the seizures are detected. This may be caused by the high number of windows. If the number of windows is too high the length of the period they cover might exceed the length of the tonic phase for some patients, and thereby cause detection to fail. The mean latency is longer for detections based only on the anterior tibial muscles compared to those based on the deltoid muscle. However for some patients latency is lower than for 
This article has been accepted for publication in a future issue of this journal, but has not been fully edited. Content may change prior to final publication.

TABLE III

THE RESULTS FOR VALIDATION OF THE TRAINED PARAMETERS (SEE TABLE II), BASED ON A SINGLE MUSCLE OR THE COMBINATION OF TWO.

\begin{tabular}{|c|c|c|c|c|c|c|c|c|c|c|}
\hline & \multirow[b]{2}{*}{ Patient } & \multicolumn{3}{|c|}{ Deltoid } & \multicolumn{3}{|c|}{ Anterior Tibial } & \multicolumn{3}{|c|}{ Combined } \\
\hline & & SEN [\%] & $\operatorname{FDR}[/ \mathrm{h}]$ & LAT [s] & SEN [\%] & FDR $[/ h]$ & LAT [s] & SEN [\%] & $\operatorname{FDR}[/ \mathrm{h}]$ & LAT $[\mathrm{s}]$ \\
\hline \multirow{3}{*}{$\begin{array}{l}\vec{\Xi} \\
\text { ठ் }\end{array}$} & 2 & 100 & 0.03 & 8.38 & 0 & 0.01 & - & 100 & 0.01 & 4.88 \\
\hline & 6 & 100 & 0.04 & 7.75 & 100 & 0.03 & 6.75 & 100 & 0.03 & 4.25 \\
\hline & 11 & 100 & 0.00 & 6.00 & 100 & 0.84 & 7.25 & 100 & 0.02 & 3.33 \\
\hline \multirow{3}{*}{ 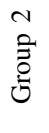 } & 1 & 100 & 0.00 & 9.00 & 100 & 0.06 & 6.75 & 100 & 0.00 & 6.00 \\
\hline & 4 & 100 & 0.09 & 11.25 & 100 & 0.09 & 19.25 & 100 & 0.00 & 14.75 \\
\hline & 9 & 100 & 0.03 & 34.44 & 100 & 0.00 & 27.44 & 100 & 0.00 & 26.31 \\
\hline \multirow{3}{*}{ 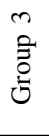 } & 5 & 100 & 0.04 & 7.13 & 50 & 0.05 & 11.75 & 100 & 0.19 & 5.75 \\
\hline & 7 & 100 & 0.03 & 17.00 & 100 & 0.11 & 14.75 & 100 & 0.00 & 9.75 \\
\hline & 10 & 100 & 0.14 & 7.17 & 67 & 0.31 & 24.88 & 100 & 0.02 & 13.00 \\
\hline \multirow{3}{*}{ 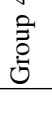 } & 3 & 100 & 0.00 & 10.25 & 0 & 0.68 & - & 100 & 0.01 & 9.75 \\
\hline & 8 & 100 & 0.00 & 12.33 & 100 & 0.32 & 11.08 & 100 & 0.16 & 7.33 \\
\hline & Mean & 100 & 0.04 & 13.66 & 77 & 0.20 & 14.11 & 100 & 0.04 & 9.85 \\
\hline
\end{tabular}
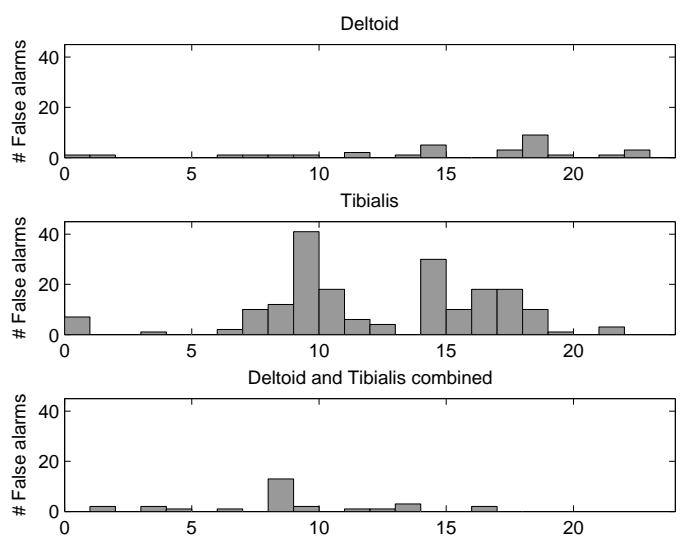

Fig. 4. Histograms of the false alarms for all 11 patients. The abscissa shows the time of the day (24-hours).

the deltoid muscle and for some patients it is higher. For all those with a lower latency all seizures are detected. The same pattern is seen for the FDR, where the mean is much higher for the results on the anterior tibial muscle compared to the deltoid muscle, but for three patients it is actually lower.

If the two sets of data are combined the results improve somewhat regarding latency, as expected. Similarly to the results based on the deltoid muscle alone, all seizures are detected, the mean FDR is low, but the mean latency is even shorter for the detection based on both muscles. However for two patients (4 and 10) the latencies are increased for the combination of both muscles as compared to the deltoid muscle alone. The explanation may be found in the fact that the latency is very high for these patients, when only the anterior tibial data are used, which implies that the seizures are seen later in this muscle than in the deltoid muscle.

\section{DISCUSSION}

The different results for the two muscles and the combination of both are not only dependent on the chosen muscle, but also on the chosen parameters, see Table II. Thus for the combined method the number of windows for a detection is low, which explains the short latency and for the anterior tibial muscle the number of windows is large, which gives long latencies. Looking at the parameters in Table II, one will see that they are more alike for the combination of the two muscles, than for the deltoid muscle alone, where group three is tested with parameters somewhat different from the others. If group three had instead been tested with parameters more alike to the other three groups (19 windows and a threshold of 241), the sensitivity would have stayed the same, but the latency would have been longer and the FDR smaller, which would bring the mean FDR to 0.03 and the mean latency to 13.9 for the deltoid muscle data alone. This suggests that an equivalent well result as presented in Table III would be obtained by using the exact same parameters for all patients.

Since combining the deltoid data with the anterior tibial data only improves the latency (by $4 \mathrm{~s}$ on average), a detection system would more appropriately be based on the deltoid muscle alone, since the gain of adding data from an extra muscle is too small. If data should be combined from two muscles in a detection system one would probably choose two muscles closer together, than the two we have used in this study.

The sensitivity and specificity of seizure detection systems based on EEG signals vary widely: 70-100\% (for sensitivity) and $0.5-72$ false detections / 24 hours (for specificity)[24], [25], [26], [27]. The best performing ones are based on invasive recordings (intracranial electrodes) or many scalp electrodes (>60)[27]. In the best of these studies they achieve the same sensitivity as our approach, but a lower FDR and shorter latency. It should, however, be considered, that their system would not be feasible for a long-term monitoring in the patients home or in the everyday life of a patient. In spite 
of using signals from a non-invasive recording (sEMG) and just one channel, we obtained a sensitivity of $100 \%$ and a specificity of 1 false-detection / 24 hours. This is compatible with the best performing EEG-based systems, but our system is easier implemented, because it is non-invasive.

If we compare our deltoid results to other studies [12], [13] who have developed an algorithm to detect tonic-clonic seizures based on motion-data, ours are more sensitive (SEN $=100 \%$ versus $88 \%[12]$ and $91 \%[13])$. The study by Kramer et al. [13] includes 15 patients (22 seizures as in our study), whereas the results by Lockman et al. [12] is based on a very limited database ( 6 patients with 8 seizures). At the same time our algorithm captures seizures in the tonic-phase, whereas the other methods focus on the clonic-phase. Therefore our latencies are shorter than in these two studies with accelerometers. We are not able to compare the FDR to [12], since they have not listed how long their recordings were, but only that they captured 204 false alarms for the 8 patients. They do, however, state that they have a large false detection rate, so we would expect their system to have a larger FDR, than what we are able to provide. Kramer et al. [13] reports an FDR of 0.004 ( 8 false alarms on 1692 hours), which is lower than what we have provided, but this should be held up against the lower sensitivity they achieved, which we find is more important to keep as close to $100 \%$ as possible.

Very few time periods were excluded from the evaluation of data, but in a real time situation it is important, that all data is useful. That means that in real time the electrode(s) collecting the data for the algorithm must be extra adhesive, so as they will not become loose. In periods where the electrode(s) are loose or have completely fallen off, the algorithm will not be able to detect any seizures.

The electrodes used to collect the data are wired; the impedances are kept low by the healthcare personal monitoring the signals and making sure that the background noise does not increase too highly. More than $96 \%$ of the data are used. In a home situation wireless-electrodes, firmly attached by a plaster specifically designed for this purpose (Ictalcare $A / S$, Denmark) would be used. The next step in our process is to implement the algorithm into the hardware of a device with such a wireless-electrode.

Other muscles might be used as well. These two muscles were chosen since, in our experience, the deltoid muscle is always strongly involved in generalised tonic-clonic seizures. Anterior tibial muscle provides a less visible placement for a detection device, if it should be worn in daytime situations.

To the best of our knowledge this is the first seizure detection algorithm based solely on the sEMG modality. We have developed a generic (the same algorithm/parameters are used for all patients) seizure detection system that is noninvasive (based on sEMG recordings), feasible (was applicable in all recruited patients), with high sensitivity (100\%), low rate of false alarms (1 / 24 hours) and able to run in real-time. The algorithm was evaluated with a 4-fold cross-validation on one or two channels of sEMG from the deltoid or anterior tibial muscle from 11 patients with tonic-clonic seizures. It can only detect one seizure type: the tonic-clonic ones. Nevertheless it is the group of patients with this seizure type that has the highest risk for injuries following seizures and SUDEP (Sudden Unexpected Death in Epilepsy Patients)[28]. Implemented in a portable device, the algorithm presented provide advantages over the alarm devices used today, based on accelerometers in a wristband or a bedalarm.

\section{CONCLUSION}

We have developed a generic seizure detection algorithm, which is the first of its kind to be based on sEMG data alone. The algorithm focuses on detection of tonic-clonic seizures as compared to normal activity. Our algorithm was validated with a 4-fold cross validation and we found that it is highly sensitive, with low false detection rate and short detection latency. For one muscle alone (deltoid) our method performed with a sensitivity of $100 \%$, a median latency of 13.7 seconds and a median FDR of $0.04 / \mathrm{h}$ corresponding to one false alarm in 24-hours. The algorithm performs well enough to be implemented in clinical practice. A first implementation in a nighttime only device would provide a median FDR of approximately one in ten days.

\section{ACKNOWLEDGMENT}

This work was supported by the Peter \& Jytte Wolf Foundation for Epilepsy, the Danish Epilepsy Centre, the Danish Agency of Science Technology and Innovation, the Technical University of Denmark, and the Danish National Advanced Technology foundation.

We are grateful to Professor Dario Farina for the fruitful discussions on this topic and for his comments and suggestions.

\section{REFERENCES}

[1] R. Fisher, W. Boas, W. Blume, C. Elger, P. Genton, P. Lee, and J. Engel Jr, "Epileptic seizures and epilepsy: definitions proposed by the International League Against Epilepsy (ILAE) and the International Bureau for Epilepsy (IBE),' Epilepsia, vol. 46, no. 4, pp. 470-472, 2005.

[2] J. Engel Jr, S. Wiebe, J. French, M. Sperling, P. Williamson, D. Spencer, R. Gumnit, C. Zahn, E. Westbrook, and B. Enos, "Practice parameter: temporal lobe and localized neocortical resections for epilepsy," Epilepsia, vol. 44, no. 6, pp. 741-751, 2003.

[3] L. Nashef and P. Ryvlin, "Sudden unexpected death in epilepsy (SUDEP): update and reflections," Neurologic clinics, vol. 27, no. 4, pp. 1063-1074, 2009.

[4] J. Hughes, "A review of sudden unexpected death in epilepsy: prediction of patients at risk," Epilepsy \& Behavior, vol. 14, no. 2, pp. 280-287, 2009.

[5] I. Conradsen, S. Beniczky, P. Wolf, D. Terney, T. Sams, and H. Sorensen, "Multi-modal intelligent seizure acquisition (MISA) system - a new approach towards seizure detection based on full body motion measures," in Engineering in Medicine and Biology Society, 2009. EMBC 2009. Annual International Conference of the IEEE. IEEE, 2009, pp. 25912595.

[6] I. Conradsen, S. Beniczky, P. Wolf, J. Henriksen, T. Sams, and H. Sorensen, "Seizure onset detection based on a Uni-or Multi-modal Intelligent Seizure Acquisition (UISA/MISA) system," in Engineering in Medicine and Biology Society (EMBC), 2010 Annual International Conference of the IEEE. IEEE, 2010, pp. 3269-3272.

[7] S. Patel, C. Mancinelli, A. Dalton, B. Patritti, T. Pang, S. Schachter, and P. Bonato, "Detecting epileptic seizures using wearable sensors," in Bioengineering Conference, 2009 IEEE 35th Annual Northeast. IEEE, 2009, pp. 1-2.

[8] T. Sorensen, U. Olsen, I. Conradsen, J. Henriksen, T. Kjaer, C. Thomsen, and H. Sorensen, "Automatic epileptic seizure onset detection using matching pursuit: A case study," in Engineering in Medicine and Biology Society (EMBC), 2010 Annual International Conference of the IEEE. IEEE, 2010, pp. 3277-3280. 
[9] E. Chua, K. Patel, M. Fitzsimons, and C. Bleakley, "Improved patient specific seizure detection during pre-surgical evaluation," Clinical Neurophysiology, vol. 122, no. 4, pp. 672-679, 2011.

[10] M. Arab, A. Suratgar, and A. Ashtiani, "Electroencephalogram signals processing for topographic brain mapping and epilepsies classification," Computers in Biology and Medicine, vol. 40, no. 9, pp. 733-739, 2010.

[11] J. Jeppesen, S. Beniczky, A. Fuglsang-Frederiksen, P. Sidenius, and Y. Jasemian, "Detection of epileptic-seizures by means of power spectrum analysis of heart rate variability: A pilot study," Technology and Health Care, vol. 18, no. 6, pp. 417-426, 2010.

[12] J. Lockman, R. Fisher, and D. Olson, "Detection of seizure-like movements using a wrist accelerometer," Epilepsy \& Behavior, 2011.

[13] U. Kramer, S. Kipervasser, A. Shlitner, and R. Kuzniecky, "A novel portable seizure detection alarm system: Preliminary results," Journal of Clinical Neurophysiology, vol. 28, no. 1, p. 36, 2011.

[14] T. Nijsen, J. Arends, P. Griep, and P. Cluitmans, "The potential value of three-dimensional accelerometry for detection of motor seizures in severe epilepsy," Epilepsy \& Behavior, vol. 7, no. 1, pp. 74-84, 2005.

[15] T. Nijsen, R. Aarts, J. Arends, and P. Cluitmans, "Automated detection of tonic seizures using 3-d accelerometry," in 4th European Conference of the International Federation for Medical and Biological Engineering. Springer, 2009, pp. 188-191.

[16] K. Cuppens, L. Lagae, B. Ceulemans, S. Van Huffel, and B. Vanrumste, "Detection of nocturnal frontal lobe seizures in pediatric patients by means of accelerometers: A first study." in Conference proceedings:31st Annual International Conference of the IEEE Engineering in Medicine and Biology Society., vol. 1, 2009, pp. 6608-6611.

[17] G. Becq, S. Bonnet, L. Minotti, M. Antonakios, R. Guillemaud, and P. Kahane, "Collection and exploratory analysis of attitude sensor data in an epilepsy monitoring unit," in Engineering in Medicine and Biology Society, 2007. EMBS 2007. 29th Annual International Conference of the IEEE. IEEE, 2007, pp. 2775-2778.

[18] _ - "Classification of epileptic motor manifestations using inertial and magnetic sensors," Computers in Biology and Medicine, 2010.

[19] I. Conradsen, S. Beniczky, K. Hoppe, P. Wolf, T. Sams, and H. Sorensen, "Seizure Onset Detection Based on One sEMG Channel," in Engineering in Medicine and Biology Society (EMBC), 2011 Annual International Conference of the IEEE. IEEE, 2011, pp. 7715-7718.

[20] I. Conradsen, P. Wolf, T. Sams, H. Sorensen, and S. Beniczky, "Patterns of muscle activation during generalized tonic and tonic-clonic epileptic seizures," Epilepsia, 2011.

[21] M. van Putten, T. Kind, F. Visser, and V. Lagerburg, "Detecting temporal lobe seizures from scalp eeg recordings: a comparison of various features," Clinical neurophysiology, vol. 116, no. 10, pp. 2480-2489, 2005.

[22] S. Zandi, R. Tafreshi, M. Javidan, and G. Dumont, "Predicting temporal lobe epileptic seizures based on zero-crossing interval analysis in scalp eeg," in Engineering in Medicine and Biology Society (EMBC), 2010 Annual International Conference of the IEEE. IEEE, 2010, pp. 55375540.

[23] M. Stone, "Cross-validatory choice and assessment of statistical predictions," Journal of the Royal Statistical Society. Series B (Methodological), vol. 36, no. 2, pp. 111-147, 1974.

[24] J. Gotman, "Automatic recognition of epileptic seizures in the eeg," Electroencephalography and clinical Neurophysiology, vol. 54, no. 5, pp. 530-540, 1982.

[25] I. Osorio, M. Frei, J. Giftakis, T. Peters, J. Ingram, M. Turnbull, M. Herzog, M. Rise, S. Schaffner, R. Wennberg et al., "Performance reassessment of a real-time seizure-detection algorithm on long ecog series," Epilepsia, vol. 43, no. 12, pp. 1522-1535, 2002.

[26] Y. Khan and J. Gotman, "Wavelet based automatic seizure detection in intracerebral electroencephalogram," Clinical Neurophysiology, vol. 114, no. 5, pp. 898-908, 2003.

[27] G. Minasyan, J. Chatten, M. Chatten, and R. Harner, "Patient-specific early seizure detection from scalp eeg," Journal of clinical neurophysiology: official publication of the American Electroencephalographic Society, vol. 27, no. 3, p. 163, 2010.

[28] R. Kloster and T. Engelskjøn, "Sudden unexpected death in epilepsy (sudep): a clinical perspective and a search for risk factors," Journal of Neurology, Neurosurgery \& Psychiatry, vol. 67, no. 4, p. 439, 1999.

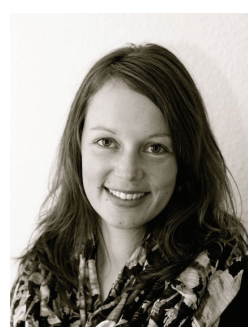

Isa Conradsen received the M.Sc. degree in biomedical engineering from the Technical University of Denmark and the University of Copenhagen, Denmark in 2008. She is currently pursuing a Ph.D. degree in the Department of Electrical Engineering, Technical University of Denmark. Her Ph.D. project is carried out in close collaboration with the Danish Epilepsy Centre, Dianalund, Denmark. Her research interests include biomedical signal processing, epilepsy, classification and pattern recognition.

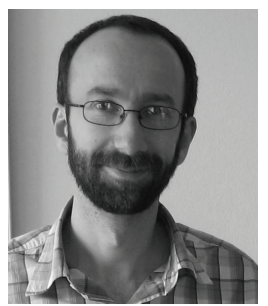

Sándor Beniczky graduated from the medical school in 1997, and he received Ph.D. degree in neuroscience in 2004 at the University of Szeged Hungary. $\mathrm{He}$ is a board certified neurologist and clinical neurophysiologist (both in Hungary and in Denmark). His main research interests are evoked potentials, source localisation, EEG and epilepsy.

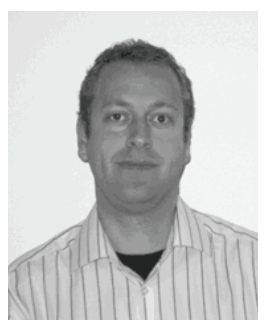

Karsten Hoppe received the M.Sc. degree in Electrical Engineering from the Technical University of Denmark in 1995 . He is currently working as Application Specialist in the ePatch group at DELTA. His main interest is ePatch based medical device design.

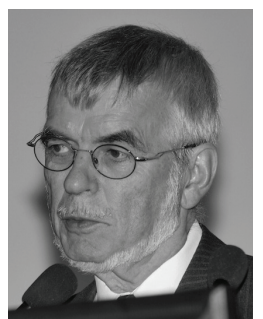

Peter Wolf is as a retired Professor of Neurology affiliated to the Danish Epilepsy Center Dianalund. His research has addressed many aspects of clinical epileptology and is at present focused on reflex epilepsies, epilepsia partialis continua, and the cultural history of epilepsy. He is involved in various international education programmes and has held numerous honorary positions in the field of epilepsy. $\mathrm{He}$ is Immediate Past President of the International League against Epilepsy.

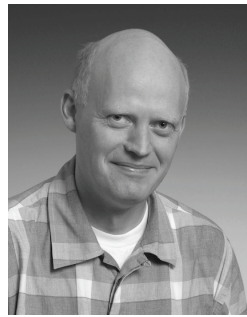

Helge B.D. Sorensen (M'90) received his M.Sc.E.E degree in Electrical Engineering and the Ph.D. degree in Electrical Engineering from Institute of Electronic Systems, Aalborg University, in 1985 and 1992 , respectively. He was initially research assistant here and from 1989-1993, he was Assistant Professor at Institute of Electronic Engineering, Aalborg University. From 1993-1995, he was Associate Professor at Engineering Academy Denmark. Currently (since 1995) he is Associate Professor and Head of the Biomedical Signal Processing research group at the Department of Electrical Engineering, Technical University of Denmark. 